\title{
Do Market-Level Hospital and Physician Resources Affect Small Area Variation in Hospital Use?
}

\author{
Jeffrey A. Alexander \\ University of Michigan School of Public Health \\ Shoou-Yih D. Lee \\ University of Illinois-Chicago \\ John R. Griffith \\ Stephen S. Mick \\ Xihong Lin \\ Jane Banaszak-Holl \\ University of Michigan School of Public Health
}

\begin{abstract}
This study evaluates the effect of market-level physician and hospital resources on hospital use. It is anticipated that higher hospital discharges are associated with (1) greater hospital and physician resources, (2) more differentiated hospital and physician resources, and (3) higher levels of teaching intensity in the community. Data on 14 modified diagnostically related groups (DRGs) and 58 hospital market communities in Michigan are analyzed during a 7-year period. Findings indicate that physician resources, hospital resources, differentiation of hospital and physician resources, and teaching intensity contribute only modestly to discharges, holding constant the socioeconomic attributes of the community and adjusting for the variation in hospital use over time. With the inclusion of hospital and physician resource variables, socioeconomic factors remain important determinants of the variation across market communities. Findings are discussed in terms of their implications for health care organizations, managed care programs, and cost control efforts in general.
\end{abstract}

Substantial attention in health services research has been given to small area variation (SAV), the observed variation in hospital use among communities, as one potential area to lower health care costs and to improve quality of

(9) Medical Care Research and Revie 1999 Sage Publications, Inc.

94 
care (Goodman and Green 1996). As noted by others, much of the variation in hospital expenditures is due to differences in admission rates rather than in lengths of stay or the amount of care provided per case (Folland and Stano 1990). Thus, if hospital admissions can be decreased, health care costs may also decline. Such area-based orientations to utilization of health services are drawing the attention of managed care organizations as they begin to adopt more population-focused planning and interventions to control costs and improve health status (e.g., Joseph et al. 1998).

Although SAV analysis has grown more sophisticated in specifying the nature and extent of variation (Volinn et al. 1994), and more practical in its application across data sources (Green 1996), less progress has been made in explaining why variation is observed across communities. Early studies inferred that physician characteristics such as physician practice style were a major factor (Wennberg 1984; Wennberg and Gittelsohn 1982). Unfortunately, the few studies that have attempted to test the practice style explanation have been fraught with methodological shortcomings (Stano 1991, 1986). A more recent line of inquiry in SAV has been based on analyses that suggest that ready access to, and supply of, acute care facilities and modern medical technology in a market may affect the culture of medical practice and physician behavior, thereby significantly increasing the use of medical services (Dartmouth Center for the Evaluative Clinical Sciences 1998). To date, however, such market structure explanations have not been extensively examined in the literature.

\section{NEW CONTRIBUTION}

This article examines the variation in hospital use among Michigan hospital market communities (HMCs), defined by geographic patterns of hospital use. We hypothesize that structural characteristics of hospitals and/or physician resources such as size, scope of services, medical specialty composition, level of hospital-based outpatient activity, and clinical support capacity of local hospitals are associated with the amount of hospital use in HMCs, over and above other characteristics of these communities that might induce demand for hospital services. This allows us to investigate whether hospital and physician resource characteristics exercise independent effects on hospital use or whether such effects are primarily a function of "demand" factors created by the socioeconomic mix of the community.

Support for this study was received from the Blue Cross Blue Shield of Michigan Foundation. This article, submitted to Medical Care Research and Review on November 4, 1997, was revised and accepted for publication on October 8, 1998. 
In contrast to previous SAV studies, we examine multiple clinical conditions and use a longitudinal research design and Poisson regression to better assess the dynamics among hospital and physician resources, socioeconomic attributes of the community, and hospital use. These methods allow us to adjust for the annual variation in hospital use, to account for depletion in clinical conditions, and to specify differences in hospital use patterns across clinical conditions, thus providing a more reliable estimation of the independent effects of hospital and physician resource characteristics and socioeconomic factors on hospital use.

\section{SUMMARY OF SIMILAR WORK}

SAV studies have attracted growing attention for more than 25 years because they show that nearby, ostensibly similar communities have substantially different rates of hospital use (Griffith et al. 1981; Paul-Shaheen, Clark, and Williams 1987; Wennberg and Gittelsohn 1973). High use is not necessarily associated with inappropriate use (Restuccia et al. 1996; Green and Becker 1994), but the variation itself remains and may have serious implications in terms of both cost and quality (Roos et al. 1995; Kuhn, Hartz, and Baras 1995; Kovac, Christie, and Bindbeutel, 1991; Tape et al. 1991). Such variation has often been attributed to "practice style," or the practice habits of physicians (Wennberg 1984). There is widespread belief that insofar as variation represents overuse or underuse of health services in certain areas and such variation arises from different provider practices, educational and corrective policies directed at local provider groups will be able to reduce unnecessary services and produce more effective and consistent levels of care (Iglehart 1984; Lundberg and Wennberg 1997).

However, several studies have shown the early assumptions about practice style to be incomplete. First, the sources of variation appear to differ by disease (McMahon et al. 1993; Gittelsohn, Halpern, and Sanchez 1991). McMahon et al. (1993) examined 112 disease groups in Michigan, concluding that community characteristics-including education, poverty, and unemploymenthave a statistically significant but differential association with observed small area hospital discharge rates for many diagnostically related groups (DRGs). Similarly, Gittelsohn and Powe (1995) documented different utilization patterns for discretionary and nondiscretionary use in Maryland.

A second finding emerging from recent SAV research is the importance of socioeconomic context. Adjustments for socioeconomic factors are now widely used in SAV studies (Gottlieb, Beiser, and O'Connor 1995; Green and Becker 1994; Escarce 1993; Stano 1991). Komaromy et al. (1996) concluded that community socioeconomic factors are more important than physician 
practice style in explaining variation in admission rates for chronic medical conditions. Surgical procedures have also been shown to vary significantly within urban areas by population ethnicity and socioeconomic status (Carlisle et al. 1995).

Access and the supply of various clinical resources have also been found to contribute to SAV. Gittelsohn and Powe (1995) found that hospital use is related to medical resources and access, as well as demography, morbidity, and physician practice patterns. Bindman et al. (1995) recognized the importance of perception of access to care in explaining use rates, concluding that communities where people perceive poor access to medical care have higher rates of hospital use for chronic diseases. Goodman et al. (1994) noted that both supply and kind of medical care are important influences on the likelihood of hospital use for pediatric medical conditions for which outpatient alternatives are available. Billings et al. (1993) also identified the importance of outpatient care alternatives. For conditions that they identified as ambulatory care sensitive, hospital use was lower in areas where appropriate outpatient care was more readily available.

Besides the emerging understanding of the determinants of SAV, there are also recent advances in the methodology used for SAV analysis. Cain and Diehr (1992) noted that small numbers are subject to large random variation and may distort findings in conventional regression analysis. When diseasespecific rates are calculated for hospital use in the typical small areas, annual counts are often low and can be expected to vary widely as a result. Several authors have proposed methods to deal with the small-numbers issue (Shwartz et al. 1994; Luft and Brown 1993; Malec and Sedransk 1993). Cain and Diehr (1992) also noted issues in measuring events that can only occur once, for example, hysterectomy. A long history of high use may deplete the population at risk, so that a point-in-time estimate would be inaccurately low.

In summary, current understanding of SAV suggests that it is still an important issue; no work has fully explained the phenomenon. It is clear that SAV relates in some way to the socioeconomic characteristics of the community and that relatively complex analytic techniques are necessary to overcome various methodological problems. SAV analysis must address specific disease entities, adjust for socioeconomic characteristics of the small areas, and include factors that approximate the supply or access to care. Methodologically, it must accommodate random variation due to small numbers of events and recognize the depletion problem.

This study attempts to address some of these issues. We examine the effects of market-level hospital and physician resource structure on hospital use in the market, using a model that accommodates market-level socioeconomic factors, and aggregates data during several years to avoid the distortions 
arising from small numbers. We examine 14 common causes for hospital use, only one of which is subject to the depletion phenomenon.

\section{CONCEPTUAL FRAMEWORK AND HYPOTHESES}

The conceptual framework for this study derives from the literature on the structure of organizational sets or networks (Evan 1967; Fennell 1980, 1982; Turk 1977). The underlying premise of this literature is that a local community of hospitals and/or physicians may influence the practice of medicine independently of the influence of any one provider, organization, or group.

This conceptual approach emphasizes the identification of clusters or communities of frequently interacting organizations, as well as, in certain cases, the "linking pin" organization that facilitates interactions among community members (Sofaer and Myrtle 1991). Most studies of interorganizational relations in health care have addressed purposeful interactions among organizations such as alliances or hospital systems. However, members of a community of health care providers might be affected in more subtle, normative fashion by the characteristics of other members, as well as the structural characteristics of the community as a whole (Fennell 1980). We follow this line of research by focusing on the effects of hospital and physician resource structure and socioeconomic characteristics of hospital market communities. This framework has particular importance in light of the frequent criticism that SAV studies are subject to the ecological fallacy. That is, many SAV studies assess relationships at the aggregate level but are based on theoretical processes occurring among individuals, for example, patients and physicians (Folland and Stano 1990). By contrast, for many procedures in high-variation DRG categories, population-based differences may have less to do with patients or their physicians but more to do with such market characteristics as availability of alternative delivery sites (Thomas, Griffith, and Durance 1980).

The study focuses specifically on market-level predictors of hospital use and develops corresponding levels of measurement for independent and dependent variables. A market area in this study is defined as a HMC or a geographically connected area of populations served by a common clusters of hospitals (Thomas, Griffith, and Durance 1980). We expect that the structure of both physician and hospital resources in HMCs will vary, both in capacity and differentiation. For hospitals within a particular HMC, this may imply differences in the number of hospitals, the average number of beds per hospital, the range of facilities provided by the cluster of hospitals, and the extent of service duplication in the HMC. Specifically, three dimensions of hospital and physician resources will be considered in the study. These dimensions, encompassing variables specific to both hospital and physician resources, 
include (1) resource capacity, (2) resource differentiation, and (3) teaching intensity.

\section{HOSPITAL AND PHYSICIAN RESOURCE CAPACITY}

This dimension assesses aggregate or average hospital and physician resource capacity or service level in an HMC. Variables represented under this dimension are measured by either (1) the average across all hospitals and/or physicians in the HMC (e.g., the mean level of hospital outpatient activity), (2) the extent to which a specific physician group in the HMC (e.g., the number of general surgeons), or (3) the amount of hospital or physician resources per unit of population in the HMC (e.g., hospital beds per 1,000).

It is expected that high levels of hospital and/or physician resources will lead to high hospital use rates. This expectation rests on two arguments. First, as some health economists have argued, greater supply of physicians is likely to produce higher demand for care mainly because of physicians' expectation to meet a target income and their ability to control medical information (Rice 1983). Second, supply of hospitals and/or physicians increases the frequency of patient referral, which, in turn, leads to high utilization of health services, including hospital use in HMCs (Dartmouth Center for the Evaluative Clinical Sciences 1998; Hine et al. 1996). For example, a recent analysis of health services use in the United States conducted by the Dartmouth Center for the Evaluative Clinical Sciences (1998) revealed a general pattern that ready access to acute care services and modern medical technology may affect the culture of medical practice and the recommendation of individual physicians, thereby significantly increasing the use of medical services.

Hypothesis 1: The greater the provider capacity in an HMC, the higher the level of hospital use.

\section{HOSPITAL AND PHYSICIAN RESOURCE DIFFERENTIATION}

This dimension assesses the degree of similarity or difference among hospital and/or physician resources in the same community. Unlike hospital and physician resource capacity, differentiation is concerned less with how many or at what level services are provided in an HMC and more with the extent of heterogeneity (or, conversely, similarity) among hospitals and/or physicians. Differentiation on selected hospital and physician resource characteristics is measured either by (1) an index of concentration/dispersion such as the diversity index of physician specialties (Teachman 1980), (2) the proportional 
representation of a hospital or physician resource group (e.g., percentage surgical specialists in an HMC), or (3) the coefficients of variation (e.g., coefficients of variation [CVs] of outpatient activity among HMC hospitals).

A central mechanism that differentiation increases hospital use is through the reduction of coordination and control. By this we mean that highly variegated HMC structures will have multiple sources of, and norms for, potential hospitalization. As a result, points of control will be diffuse and numerous, thwarting a simple, easily identifiable way to identify, to monitor, and to enforce strict utilization controls (Scott 1992). Furthermore, lack of professional consensus and hospitalization criteria may also promote inappropriate and overuse of hospital services (Hermann et al. 1995).

A second reason why differentiation influences high use is the fragmentation of activity among specialized hospitals and/or physicians. This fragmentation, in turn, increases hospital use because services such as diagnostic tests tend to be repeated by different types of specialists (Luft et al. 1986).

Hypothesis 2: The more differentiated the HMC hospital and physician resources, the higher the level of hospital use.

\section{TEACHING INTENSITY}

Teaching intensity captures the level of tertiary care activity and its potential impact on general hospital use in the HMC (Fennell 1980). Hospitals in which intensive teaching is performed may act as institutional leaders dictating hospital use in neighboring facilities through referral arrangements or the like (Wyatt et al. 1997). As such, we expect that the rates of hospital use will be higher in markets with higher levels of teaching activity because of the tendency of teaching facilities to emphasize inpatient care, technology, and specialization in diagnosis and treatment.

Hypothesis 3: The greater the level of teaching intensity in an HMC, the higher the level of hospital use.

\section{METHOD}

\section{RESEARCH DESIGN AND SAMPLE}

The study adopts a longitudinal panel design. We analyze changes in hospital use in 58 hospital market communities in the lower peninsula of Michigan from 1986 to 1992. An HMC is identified by assigning zip codes to a hospital or a hospital group on the basis of where the plurality of residents of a zip 
code area seek hospital care. Each zip code area in Michigan's lower peninsula is assigned uniquely to an HMC. Two of the original $60 \mathrm{HMC}$ are collapsed into adjacent communities because no zip code in the HMC contributes more than 50 percent of its admissions to the hospitals in the HMCs (Thomas, Griffith, and Durance 1980).

\section{DATA SOURCES}

Data are obtained from four sources: (1) 1990 population census, (2) 19861992 American Hospital Association (AHA) annual hospital surveys, (3) 19861992 Michigan Inpatient Data Base (MIDB), and (4) 1986 and 1990 Michigan Physician Censuses (MPCs). The 1990 census provides demographic and socioeconomic information for each zip code area in Michigan. We aggregate this information to construct the total population, socioeconomic status, age, and ethnic composition for each HMC. The AHA surveys provide information on hospital bed capacity, services, and medical staff and other personnel.

MIDB contains more than 95 percent of annual discharges from Michigan short-term hospitals, with identifiers for patient age, gender, ethnicity, zip code of residence, and up to seven diagnoses and four surgical procedures. The diagnoses are used to assign DRGs using the standard Health Care Financing Administration (HCFA) classification system. The DRGs of the HCFA are then collapsed into 112 modified DRGs (MDRGs) suggested by Wennberg, Freeman, and Culp (1987).

The 1986 and 1990 MPCs were part of the medical licensure program in Michigan (Office of Health and Medical Affairs 1990). The census includes information on the physician's practice type, specialty, board certification, and time spent on patient care in different practice areas. Counts of physicians exclude nonpracticing physicians and interns/residents. Two procedures are implemented to construct physician variables for the study. First, we adjust for physicians practicing in more than one location by allocating the fractional share of their time to each of their practice areas, identified by zip codes. For example, a physician providing patient care for 15 hours per week in one zip code, 15 hours in another, and 20 hours in yet a third contributes 0.3 full-time equivalent (FTE) physician manpower to the first two zip codes and 0.4 FTE to the third. Physician FTEs in every zip code area are then aggregated to represent the supply of physician manpower in an HMC. Second, interpolation and extrapolation are used to estimate values for 1987-1989 and 1991-1992.

Data from these sources are merged for all years to form a pooled, crosssectional time-series data file. For purposes of the analysis, counts of discharges within each MDRG are grouped according to six age categories (0-14, $15-29,30-44,45-64,65-74,75$ years or above). This permits us to adjust for the 
TABLE 1 Results of Poisson Regression Modeling: Baseline Models (unstandardized coefficients)

\begin{tabular}{|c|c|c|c|c|c|c|}
\hline$M D R G^{\mathrm{a}}$ & $\begin{array}{c}19 \\
\text { Adult } \\
\text { Simple } \\
\text { Pneumonia }\end{array}$ & $\begin{array}{c}21 \\
\text { Adult } \\
\text { Bronchitis } \mathcal{E} \\
\text { Asthma }\end{array}$ & $\begin{array}{c}\text { Adult } \\
\text { Gastroenteritis }\end{array}$ & Chemotherapy & $\begin{array}{c}98 \\
\text { Infectious } \\
\text { Disease } \\
\text { Disorders }\end{array}$ & $\begin{array}{c}02 \\
\text { Specific } \\
\text { Cerebrovascular } \\
\text { Disorder }\end{array}$ \\
\hline INTERCEPT & $-14.18^{* * *}$ & $4.66^{* * *}$ & $-11.55^{* * *}$ & $-7.63^{* * *}$ & $-6.28^{* * *}$ & $-11.00^{* * *}$ \\
\hline $\mathrm{SES}^{\mathrm{b}}$ & $-0.16^{* * *}$ & -0.16 & $-0.15^{* * *}$ & $-0.16^{* *}$ & 0.03 & $-0.22 \mathrm{E}-2$ \\
\hline \multicolumn{7}{|l|}{ AFRICAN } \\
\hline AMERICAN & $-0.77^{* *}$ & -0.46 & $-0.99 * * *$ & -0.70 & $0.44^{*}$ & $0.40^{* * *}$ \\
\hline AGE1529 & $6.97^{* * *}$ & $-11.47^{* * *}$ & $5.39 * * *$ & -0.40 & $-1.22^{* * *}$ & $1.04^{* * *}$ \\
\hline AGE3044 & $7.62^{* * *}$ & $-10.98^{* * *}$ & $5.78^{* * *}$ & $0.65^{* * *}$ & $-1.18^{* * *}$ & $2.54^{* * *}$ \\
\hline AGE4564 & $8.46^{* * *}$ & $-10.31^{* * *}$ & $6.30^{* * *}$ & $2.40^{* * *}$ & $-0.47^{* * *}$ & $4.63^{* * *}$ \\
\hline AGE6574 & $9.38^{* * *}$ & $-9.76^{* * *}$ & $6.81^{* * *}$ & $2.92^{* * *}$ & $0.40^{* * *}$ & $5.89^{* * *}$ \\
\hline AGE75+ & $10.34^{* * *}$ & $-9.58^{* * *}$ & $7.35^{* * *}$ & $2.14^{* * *}$ & $1.21^{* * *}$ & $6.82^{* * *}$ \\
\hline BORDER & 0.08 & -0.37 & $-0.38^{*}$ & $-1.33^{* *}$ & 0.08 & $-0.10^{*}$ \\
\hline YR87 & $-0.11^{* * *}$ & $-0.09^{* * *}$ & $-0.06^{* * *}$ & 0.01 & -0.01 & 0.01 \\
\hline YR88 & $-0.09^{* * *}$ & $-0.07^{* * *}$ & $-0.10^{* * *}$ & $-0.04 \mathrm{E}-1$ & 0.03 & $-0.51 \mathrm{E}-2$ \\
\hline YR89 & $-0.07^{* * *}$ & $-0.06^{* * *}$ & $-0.13^{* * *}$ & 0.04 & $0.08^{* *}$ & 0.04 \\
\hline YR90 & $0.07 \mathrm{E}-2$ & $-0.21^{* * *}$ & $-0.22^{* * *}$ & $0.03 \mathrm{E}-1$ & $0.12^{* *}$ & $0.05^{*}$ \\
\hline YR91 & -0.03 & $-0.33^{* * *}$ & $-0.32^{* * *}$ & $-0.03 \mathrm{E}-1$ & $0.14^{* * *}$ & $0.08^{* * *}$ \\
\hline YR92 & $-0.06^{* *}$ & $-0.62^{* * *}$ & $-0.38^{* * *}$ & -0.05 & $0.08^{*}$ & $0.09^{* * *}$ \\
\hline
\end{tabular}

effect of age on hospital use by including age dummy variables in the analysis (Breslow and Day 1987). Therefore, our unit of analysis is the age-group within each HMC rather than the HMC. The final data set contains seven years of repeated observations for each of the six age categories in each of the 58 MHCs $(N=7 \times 6 \times 58=2,436)$.

\section{MEASURES}

Dependent Variable. The dependent variables are age-specific discharges within Wennberg's diagnostic groupings. Of the 112 MDRGs, 14 that display the highest frequency and coefficients of variation in discharge rate across HMCs are selected for analysis. High frequency or volume suggests the potential importance of these hospital services on cost. Large coefficients of variation suggest potentially inappropriate use of these services across HMCs. Furthermore, from an analytic standpoint, diagnostic groups with higher coefficients of variation also provide the requisite variability for exam- 


\begin{tabular}{|c|c|c|c|c|c|c|c|}
\hline $\begin{array}{c}27 \\
\text { Acute } \\
\text { Myocardial } \\
\text { Infaction }\end{array}$ & $\begin{array}{c}29 \\
\text { Heart } \\
\text { Failure E } \\
\text { Shock }\end{array}$ & $\begin{array}{c}\text { Cardiac } \\
\text { Arrhythmia }\end{array}$ & $\begin{array}{l}\text { Angina } \\
\text { Pectoris }\end{array}$ & $\begin{array}{c}25 \\
\text { Major } \\
\text { Cardiovascular } \\
\text { Operations }\end{array}$ & $\begin{array}{c}26 \\
\text { Other } \\
\text { Cardiovascular } \\
\text { Operations }\end{array}$ & $\begin{array}{c}55 \\
\text { Gallbladder } \\
\text { Disease with } \\
\text { Cholecystectomy }\end{array}$ & $\begin{array}{c}60 \\
\text { Back } \\
\text { and Neck } \\
\text { Operations }\end{array}$ \\
\hline$-12.04^{* * *}$ & $-10.69^{* *}$ & $* *-9.68^{* * *}$ & $-11.61^{* * *}$ & $-8.00^{* * *}$ & $-10.52^{* * *}$ & $-10.69^{* * *}$ & $-9.86^{* * *}$ \\
\hline-0.06 & $-0.04^{*}$ & -0.05 & $-0.10^{*}$ & $-0.01 E-1$ & 0.01 & $-0.09^{* * *}$ & -0.10 \\
\hline$-0.58^{*}$ & 0.07 & -0.37 & -0.54 & $-0.85^{* *}$ & -0.06 & $-1.13^{* * *}$ & $-2.42^{* * *}$ \\
\hline $1.78^{* * *}$ & 0.03 & $1.00^{* * *}$ & 1.43 & $-1.55^{* * *}$ & $1.08^{* * *}$ & $4.04^{* * *}$ & $2.26^{* * *}$ \\
\hline $4.68^{* * *}$ & $2.31^{* * *}$ & $2.01^{* * *}$ & $4.78^{* * *}$ & $0.12^{* *}$ & $2.85^{* * *}$ & $4.60^{* * *}$ & $3.70^{* * *}$ \\
\hline $6.64^{* * *}$ & $4.81^{* * *}$ & $3.60^{* * *}$ & $6.58^{* * *}$ & $2.49^{* * *}$ & $4.62^{* * *}$ & $5.13^{* * *}$ & $4.01^{* * *}$ \\
\hline $7.41^{* * *}$ & $6.13^{* * *}$ & $4.65^{* * *}$ & $7.18^{* * *}$ & $3.34^{* * *}$ & $5.28^{* * *}$ & $5.38^{* * *}$ & $3.93^{* * *}$ \\
\hline $7.91^{* * *}$ & $7.06^{* * *}$ & $5.24^{* * *}$ & $7.57^{* * *}$ & $2.95^{* * *}$ & $5.55^{* * *}$ & $5.23^{* * *}$ & $3.41^{* * *}$ \\
\hline$-0.20^{*}$ & -0.16 & $-0.23^{*}$ & -0.07 & $-0.30^{* * *}$ & -0.03 & $-0.16^{* * *}$ & $-0.39^{*}$ \\
\hline-0.02 & $-0.05^{* * *}$ & 0.02 & -0.01 & 0.01 & $0.14^{* * *}$ & $0.05^{* * *}$ & $0.08^{* * *}$ \\
\hline$-0.06^{*}$ & -0.02 & $0.06^{*}$ & -0.03 & $0.11^{* * *}$ & $0.20^{* * *}$ & $0.06^{* * *}$ & $0.18^{* * *}$ \\
\hline$-0.13^{* * *}$ & $0.04^{*}$ & $0.06^{*}$ & -0.02 & $0.18^{* * *}$ & $0.27^{* * *}$ & $0.04^{*}$ & $0.24^{* * *}$ \\
\hline$-0.23^{* * *}$ & $0.11^{* * *}$ & -0.01 & -0.03 & $0.21^{* * *}$ & $0.48^{* * *}$ & 0.04 & $0.34^{* * *}$ \\
\hline$-0.21^{* * *}$ & $0.16^{* * *}$ & 0.03 & $-0.07^{*}$ & $0.11^{* *}$ & $0.47^{* * *}$ & $0.09 *$ & $0.36^{* * *}$ \\
\hline$-0.19^{* * *}$ & $0.22^{* * *}$ & 0.02 & -0.04 & $0.18^{* * *}$ & $0.54^{* * *}$ & 0.09 & $0.38^{* * *}$ \\
\hline
\end{tabular}

a. $\mathrm{MDRG}=$ modified diagnostically related group .

b. SES = socioeconomic status.

${ }^{*} p<.05 .{ }^{* *} p<.01 .{ }^{* * *} p<.001$.

ining the contribution of hospital and physician resources relative to that of socioeconomic factors on hospital use. The numbers of discharges of the selected MDRGs range from 14,446 (cardiac arrhythmia) to 28,741 (heart failure and shock). The coefficient of variation across HMCs ranges from 130.30 (acute myocardial infarction) to 205.79 (major cardiovascular operations) in 1990. Ten of these 14 MDRGs are medical, diagnostic procedures and 4 are surgical operations. The 14 specific MDRGs examined in the study are listed in Tables 1 and 2.

Socioeconomic Factors. Three socioeconomic variables are analyzed: percentage of African Americans, age, and socioeconomic status of the HMC. Five dummy variables (AGE1529, AGE3044, AGE4564, AGE6574, and AGE75+) are used to control for the age effect. The $0-14$ category serves as the reference group. Socioeconomic status is a factor score based on four characteristics of the HMC: percentage of population older than 15 years with high school diploma, percentage of population below poverty, percentage of labor population unemployed, and per capita income. 
104 MCRER 56:1 (March 1999)

TABLE 2 Results of Poisson Regression Modeling: Full Models (unstandardized coefficients)

\begin{tabular}{|c|c|c|c|c|c|c|}
\hline$M D R G^{\mathrm{a}}$ & $\begin{array}{c}19 \\
\text { Adult } \\
\text { Simple } \\
\text { Pneumonia }\end{array}$ & $\begin{array}{c}21 \\
\text { Adult } \\
\text { Bronchitis } \mathcal{E} \\
\text { Asthma }\end{array}$ & $\begin{array}{l}\text { Adult } \\
\text { Gastroenteritis }\end{array}$ & Chemotherapy & $\begin{array}{c}98 \\
\text { Infectious } \\
\text { Disease } \\
\text { Disorders }\end{array}$ & $\begin{array}{c}02 \\
\text { Specific } \\
\text { Cerebrovascular } \\
\text { Disorder }\end{array}$ \\
\hline INTERCEPT & $-13.81^{* * *}$ & $-14.79^{* * *}$ & $-13.67^{* * *}$ & $-7.90^{* * *}$ & $-6.16^{* * *}$ & $-11.03^{* * *}$ \\
\hline $\mathrm{SES}^{\mathrm{b}}$ & $-0.05^{*}$ & $-0.11^{* *}$ & $-0.06^{*}$ & -0.10 & 0.08 & 0.02 \\
\hline \multicolumn{7}{|l|}{ AFRICAN } \\
\hline AMERICAN & $0.34^{*}$ & 0.48 & -0.24 & -0.08 & $1.08^{* * *}$ & $0.48^{* *}$ \\
\hline AGE1529 & $6.97^{* * *}$ & $7.99 * * *$ & $7.45^{* * *}$ & -0.04 & $-1.21^{* * *}$ & $1.03^{* * *}$ \\
\hline AGE3044 & $7.62^{* * *}$ & $8.48^{* * *}$ & $7.84^{* * *}$ & $0.65^{* * *}$ & $-1.18^{* * *}$ & $2.54^{* * *}$ \\
\hline AGE4564 & $8.46^{* * *}$ & $9.14^{* * *}$ & $8.37^{* * *}$ & $2.41^{* * *}$ & $-0.47^{* * *}$ & $4.62^{* * *}$ \\
\hline AGE6574 & $9.38^{* * *}$ & $9.70^{* * *}$ & $8.88^{* * *}$ & $2.93^{* * *}$ & $0.40^{* * *}$ & $5.88^{* * *}$ \\
\hline AGE75+ & $10.34^{* * *}$ & $9.87^{* * *}$ & $9.41^{* * *}$ & $2.15^{* * *}$ & $1.22^{* * *}$ & $6.81^{* * *}$ \\
\hline BORDER & -0.04 & -0.33 & -0.34 & -1.28 & 0.02 & -0.08 \\
\hline YR87 & $-0.11^{* * *}$ & $-0.10^{* * *}$ & $-0.07^{* * *}$ & -0.01 & 0.02 & $0.03^{*}$ \\
\hline YR88 & $-0.06^{*}$ & $-0.07^{*}$ & $-0.11^{* * *}$ & 0.01 & 0.05 & 0.02 \\
\hline YR89 & -0.03 & -0.05 & $-0.14^{* * *}$ & 0.06 & $0.11^{* *}$ & $0.07^{* *}$ \\
\hline YR90 & 0.04 & $-0.20^{* * *}$ & $-0.23^{* * *}$ & 0.03 & $0.15^{* * *}$ & $0.09^{* * *}$ \\
\hline YR91 & 0.02 & $-0.31^{* * *}$ & $-0.36^{* * *}$ & 0.04 & $0.17^{* * *}$ & $0.14^{* * *}$ \\
\hline YR92 & $-0.01 \mathrm{E}-1$ & $-0.60^{* * *}$ & $-0.41^{* * *}$ & -0.01 & $0.13^{*}$ & $0.17^{* * *}$ \\
\hline \multicolumn{7}{|c|}{$\begin{array}{l}\text { Part A: capacity } \\
\text { measures }\end{array}$} \\
\hline SPECPCMD & -0.03 & 0.44 & 0.07 & 0.51 & -0.04 & 0.26 \\
\hline SPECPED & $-1.28^{*}$ & -0.14 & -0.18 & 1.12 & -0.78 & $-1.12^{* *}$ \\
\hline SPECSURG & -0.38 & $-1.36^{*}$ & -0.37 & -0.28 & -0.85 & -0.22 \\
\hline SPECHOSP & -0.16 & -1.18 & -0.06 & -1.12 & $-1.56^{*}$ & -0.22 \\
\hline BED & 0.02 & -0.04 & 0.04 & 0.01 & 0.04 & 0.02 \\
\hline M_DIF & 0.37 & 0.23 & $0.24^{*}$ & 0.06 & 0.07E-1 & -0.04 \\
\hline M_RN & -0.10 & -0.31 & $0.09 \mathrm{E}-1$ & -0.21 & -0.07 & -0.12 \\
\hline M_OA & 0.02 & 0.08 & 0.16 & -0.02 & 0.09 & -0.02 \\
\hline M_PC & 0.33 & 0.27 & 0.06 & $0.67^{* *}$ & $-0.44^{*}$ & -0.08 \\
\hline \multicolumn{7}{|l|}{$\begin{array}{l}\text { Part B: diversity } \\
\text { measures }\end{array}$} \\
\hline PCMD & -0.08 & 0.19 & -0.06 & -0.25 & 0.10 & -0.27 \\
\hline DVSTY & -0.54 & 0.30 & -0.15 & 0.18 & 0.32 & 0.35 \\
\hline CV_DIF & $-0.02 \mathrm{E}-2$ & $0.03 \mathrm{E}-2$ & $0.04 \mathrm{E}-2$ & $-0.02 E-2$ & $0.04 \mathrm{E}-2$ & $0.01 \mathrm{E}-2$ \\
\hline CV_RN & $-0.01 \mathrm{E}-1$ & $0.01 \mathrm{E}-1$ & $0.06 \mathrm{E}-2$ & $-0.08 \mathrm{E}-2$ & $0.01 \mathrm{E}-1$ & $0.02 \mathrm{E}-2$ \\
\hline CV_OA & $-0.02 \mathrm{E}-2$ & $-0.05 E-2$ & $-0.08 \mathrm{E}-2$ & $0.09 \mathrm{E}-2$ & $-0.05 \mathrm{E}-2$ & $0.04 \mathrm{E}-3$ \\
\hline CV_PC & $0.02 \mathrm{E}-1^{*}$ & $-0.01 \mathrm{E}-1$ & $-0.06 \mathrm{E}-2$ & $0.02 \mathrm{E}-1$ & $0.04 \mathrm{E}-2$ & $-0.03 \mathrm{E}-2$ \\
\hline \multicolumn{7}{|c|}{$\begin{array}{l}\text { Part C: teaching } \\
\text { intensity measure }\end{array}$} \\
\hline FTERES & -0.02 & -0.08 & $-0.21^{* *}$ & -0.12 & -0.06 & 0.10 \\
\hline
\end{tabular}




\begin{tabular}{|c|c|c|c|c|c|c|c|}
\hline $\begin{array}{c}27 \\
\text { Acute } \\
\text { Myocardial } \\
\text { Infaction }\end{array}$ & $\begin{array}{l}29 \\
\text { Heart } \\
\text { Failure E } \\
\text { Shock }\end{array}$ & $\begin{array}{c}\text { Cardiac } \\
\text { Arrhythmia }\end{array}$ & $\begin{array}{l}\text { Angina } \\
\text { Pectoris }\end{array}$ & $\begin{array}{c}25 \\
\text { Major } \\
\text { Cardiovascular } \\
\text { Operations }\end{array}$ & $\begin{array}{c}26 \\
\text { Other } \\
\text { Cardiovascular } \\
\text { Operations }\end{array}$ & $\begin{array}{c}55 \\
\text { Gallbladder } \\
\text { Disease with } \\
\text { Cholecystectomy }\end{array}$ & $\begin{array}{c}60 \\
\text { Back } \\
\text { and Neck } \\
\text { Operations }\end{array}$ \\
\hline$-11.72^{* * *}$ & $-10.19^{* * *}$ & $* *-9.41^{* * *}$ & $-7.22^{* * *}$ & $-7.76^{* * *}$ & $-10.64^{* * *}$ & $-10.75^{* * *}$ & $-10.32^{* * *}$ \\
\hline-0.02 & 0.01 & $-0.08 \mathrm{E}-1$ & -0.08 & 0.05 & $0.07^{*}$ & $-0.08^{* * *}$ & $-0.12^{*}$ \\
\hline-0.05 & 0.46 & -0.16 & 0.11 & -0.41 & $0.43^{*}$ & $-0.83^{* * *}$ & $-2.03^{* * *}$ \\
\hline $1.78^{* * *}$ & 0.03 & $1.02^{* * *}$ & $-2.57^{* * *}$ & $-1.57^{* * *}$ & $1.07^{* * *}$ & $3.98^{* * *}$ & $2.15^{* * *}$ \\
\hline $4.69^{* * *}$ & $2.33^{* * *}$ & $2.04^{* * *}$ & $0.70^{*}$ & $0.12^{* *}$ & $2.83^{* * *}$ & $4.54^{* * *}$ & $3.56^{* * *}$ \\
\hline $6.65^{* * *}$ & $4.82^{* * *}$ & $3.63^{* * *}$ & $2.49^{* * *}$ & $2.50^{* * *}$ & $4.60^{* * *}$ & $5.06^{* * *}$ & $3.87^{* * *}$ \\
\hline $7.42^{* * *}$ & $6.14^{* * *}$ & $4.69^{* * *}$ & $3.09 * * *$ & $3.35^{* * *}$ & $5.26^{* * *}$ & $5.32^{* * *}$ & $3.81^{* * *}$ \\
\hline $7.93^{* * *}$ & $7.08^{* * *}$ & $5.28^{* * *}$ & $3.48^{* * *}$ & $2.96^{* * *}$ & $5.52^{* * *}$ & $5.17^{* * *}$ & $3.32^{* * *}$ \\
\hline$-0.26^{* * *}$ & -0.18 & $-0.26^{*}$ & -0.18 & -0.32 & -0.01 & $-0.13^{* *}$ & $-0.46^{* *}$ \\
\hline-0.02 & -0.01 & $0.08 \mathrm{E}-1$ & 0.02 & 0.01 & $0.14^{* * *}$ & 0.03 & 0.03 \\
\hline-0.04 & 0.02 & 0.05 & $0.07 \mathrm{E}-1$ & $0.12^{* * *}$ & $0.22^{* * *}$ & 0.03 & $0.12^{* * *}$ \\
\hline$-0.10^{* *}$ & $0.11^{* *}$ & 0.05 & 0.04 & $0.20^{* * *}$ & $0.30^{* * *}$ & -0.01 & $0.15^{* *}$ \\
\hline$-0.21^{* * *}$ & $0.18^{* * *}$ & -0.02 & 0.04 & $0.24^{* * *}$ & $0.51^{* * *}$ & -0.01 & $0.24^{* * *}$ \\
\hline$-0.18^{* * *}$ & $0.27^{* * *}$ & 0.03 & 0.05 & $0.15^{* * *}$ & $0.50^{* * *}$ & $0.02 \mathrm{E}-1$ & $0.21^{* *}$ \\
\hline$-0.16^{* *}$ & $0.35^{* * *}$ & 0.02 & 0.10 & $0.22^{* * *}$ & $0.59^{* * *}$ & $-0.08 \mathrm{E}-1$ & $0.21^{* *}$ \\
\hline $0.59^{* * *}$ & 0.08 & $0.38^{*}$ & $0.89^{*}$ & $0.34^{*}$ & 0.09 & 0.22 & -0.32 \\
\hline-1.26 & -0.83 & -0.37 & -1.78 & 0.29 & 0.76 & 0.98 & 0.78 \\
\hline-0.53 & $-0.90^{*}$ & -0.66 & $-2.30^{* *}$ & 0.64 & 0.33 & 0.21 & 0.51 \\
\hline $0.01 \mathrm{E}-1$ & -0.43 & 0.99 & -1.43 & $-1.31^{* * *}$ & -0.67 & $-1.10^{*}$ & $-1.36^{*}$ \\
\hline-0.02 & 0.02 & -0.02 & -0.03 & -0.01 & 0.04 & 0.01 & -0.02 \\
\hline 0.14 & -0.21 & -0.03 & -0.10 & $-0.21^{*}$ & -0.07 & 0.04 & 0.05 \\
\hline-0.20 & $-0.31^{*}$ & -0.22 & -0.47 & $-0.20^{*}$ & -0.12 & 0.15 & $0.51^{* *}$ \\
\hline 0.03 & -0.07 & 0.01 & -0.06 & 0.03 & -0.01 & $0.15^{* *}$ & $0.17^{* *}$ \\
\hline-0.11 & -0.31 & 0.14 & -0.44 & 0.07 & -0.18 & -0.14 & 0.08 \\
\hline$-0.46^{*}$ & -0.28 & -0.32 & -0.32 & -0.11 & 0.36 & -0.11 & 0.45 \\
\hline 0.21 & 0.24 & -0.12 & 1.17 & -0.15 & -0.22 & 0.10 & 0.25 \\
\hline $0.02 \mathrm{E}-3$ & $0.03 \mathrm{E}-2$ & $-0.04 \mathrm{E}-2$ & $0.02 \mathrm{E}-2$ & $-0.01 \mathrm{E}-2$ & $-0.02 \mathrm{E}-2$ & $-0.05 \mathrm{E}-2$ & $-0.01 \mathrm{E}-1$ \\
\hline$-0.01 \mathrm{E}-1$ & $-0.07 \mathrm{E}-4$ & $0.01 \mathrm{E}-1$ & $-0.09 \mathrm{E}-2$ & $0.01 \mathrm{E}-1$ & $0.07 \mathrm{E}-2$ & $-0.05 \mathrm{E}-2$ & $0.02 \mathrm{E}-1^{*}$ \\
\hline $0.03 \mathrm{E}-2$ & $0.08 \mathrm{E}-2$ & $-0.03 \mathrm{E}-2$ & $0.02 \mathrm{E}-1$ & $0.06 \mathrm{E}-2$ & $0.07 \mathrm{E}-2$ & $0.04 \mathrm{E}-3$ & $-0.01 \mathrm{E}-1^{*}$ \\
\hline $0.08 \mathrm{E}-2$ & $-0.08 \mathrm{E}-2$ & $-0.01 \mathrm{E}-1$ & $-0.09 \mathrm{E}-2$ & $-0.01 \mathrm{E}-1$ & $-0.02 \mathrm{E}-2$ & $0.05 \mathrm{E}-2$ & $0.02 \mathrm{E}-1$ \\
\hline-0.07 & 0.04 & 0.03 & 0.06 & -0.06 & $-0.22^{* * *}$ & $-0.20^{* *}$ & $-0.33^{* *}$ \\
\hline
\end{tabular}

Note: See text for an explanation of measures.

a. MDRG = Modified diagnostically related group .

b. SES = socioeconomic status .

${ }^{*} p<.05 .{ }^{* *} p<.01 .{ }^{* * *} p<.001$. 
Hospital and Physician Resource Structure. We base our measures of the capacity and differentiation of physician resources within HMCs on (1) medical specialty (primary care, OB/GYN \& related specialties, other medical and pediatric specialties, general surgery and surgical specialties, hospital-based specialties [e.g., pathology, radiology, anesthesiology], psychiatry and related specialties, and others) and (2) type of physician (allopathic vs. osteopathic). Osteopaths constitute a significant portion of the physician population in Michigan. Because of the differences in training and practice style between osteopaths and allopaths, we expect hospital use to vary in part as a function of the proportion of allopathic physicians in the HMC.

Hospital resources include capacity and differentiation measures of HMC hospital beds, differences in hospital services, hospital outpatient activity, registered nurse staffing, and medical staff composition. Finally, we used the number of medical interns and residents per 1,000 population to indicate the level of teaching intensity in the HMC.

A dummy variable representing HMCs bordering Indiana and Ohio is used to adjust for cross-state utilization of hospital services. We also incorporate dummy variables for the 6 years of the study (1986 serves as the reference year) to account for annual variation in hospital discharges.

Additional, detailed information on measures used in the study can be obtained, on request, from the authors.

\section{ANALYSIS}

To analyze hospital use based on count data, we employ a multivariate analog of a Poisson regression model (Breslow and Day 1987). A simplified form of the model is the following:

$$
\log \left(\mu_{i j t}\right)=\log \left(N_{\mathrm{ijt}}\right)+\beta_{0}+\beta_{1} Z_{\mathrm{j}}+\beta_{2} X_{\mathrm{ijt}},
$$

where $\mu_{i j t}$ is the expected count of discharges for the $i$ th age-group in the $j$ th HMC at time $t, N_{\mathrm{ijt}}$ the number of people in age-group $i$ in HMC $j$ at time $t, Z_{\mathrm{j}}$ is the vector of socioeconomic attributes in $\mathrm{HMC} j, X_{\mathrm{ijt}}$ is the vector of hospital and physician resource characteristics for age-group $i$ in HMC $j$ at time $t$, and $\beta$ s are the regression coefficients.

Because the repeated, time-series observations in our longitudinal design may be autocorrelated, standard regression models may yield artificially small standard errors and lead to overestimation of statistical significance (Hannan and Young 1977; Zeger and Liang 1992). We use generalized estimating equations (GEEs) to estimate Poisson regression on our correlated data (Liang and Zeger 1986; Zeger and Liang 1992). GEEs treat autocorrelation as a 
nuisance and estimate it separately from the estimation of regression coefficients. This approach generates consistent estimates of parameters under minimal assumptions of the correlation pattern.

We employ the same set of covariates for each of the 14 MDRGs to investigate if a consistent pattern exists regarding the independent effects of hospital and physician resources and socioeconomic factors on hospital use. Our hypotheses are evaluated based on nested modeling. We first enter percentage African Americans, socioeconomic status, a dummy variable indicating border HMCs, and dummy variables representing age and time as the baseline model. As a test of Hypotheses 1-3, measures of the hospital and physician resource structure are added to the baseline model to determine if these variables are significantly and independently associated with hospital use. Significance of individual hospital and physician resource variables and socioeconomic factors will be examined with Wald tests. In addition, we discuss the effects of socioeconomic variables after controlling for physician and hospital resources, because these effects may indicate market-level inequalities in hospital use not associated with hospital and physician resources.

\section{RESULTS}

\section{EFFECTS OF SOCIOECONOMIC}

\section{VARIABLES ON HOSPITAL USE}

The first set of analyses examines the effects of the two socioeconomic covariates (the socioeconomic status [SES] scale and percentage African Americans) and the control variables, including local age composition, year in study, and border HMCs on the 14 MDRGs. Results of the baseline models are reported in Table 1.

Results corroborate recent studies that have shown that these socioeconomic factors have significant effects on SAV. Of the 14 MDRGs, the level of SES and percentage African Americans in the community have significant effects on all but three of the MDRGs-adult bronchitis and asthma, cardiac arrhythmia, and other cardiovascular operations. Percentage African Americans in the community is significant for 8 of the 14 MDRGs, 5 in medical and 3 in surgical diagnoses/procedures. In 6 of the 8 cases, the percentage African Americans is associated with lower discharge rates; only for infectious disease disorders and specific cerebrovascular disorders is the percentage African Americans associated with increased discharge rates. The index of SES in the community is significant for six MDRGs and consistently has a negative association with hospital use: the higher the community's level of SES, the lower 
the market area discharge rate. This negative association holds more often for medical diagnoses: 5 of 10 effects on medical discharge rates are significant compared with 1 of 4 significant effects on surgical discharge rates. In sum, SES and percentage African Americans have significant independent effects on MDRGs when included in the same model, and these relationships are found for 10 of 14 MDRGs.

\section{TESTS OF HYPOTHESES}

We test our hypotheses by adding the physician and hospital resource variables to the baseline model. Results of these Poisson regressions are presented in Table 2.

Hypothesis 1 predicts that physician and hospital resources are positively associated with hospital use across HMCs. Physician resource capacity includes the number of FTE primary care physicians per 1,000 population (SPECPCMD), number of FTE medical and pediatric specialists per 1,000 population (SPECPED), number of FTE general surgeons and surgical specialists (SPECSURG), and number of FTE hospital-based specialists per 1,000 population (SPECHOSP). Hospital resource capacity is measured by hospital beds per 1,000 population (BED), average number of services provided by the hospital that are not available in other hospitals in the same HMC (M_DIF), average number of registered nurses per bed (M_RN), average level of hospital outpatient activity (M_OA), and average percentage of primary care physicians on staff (M_PC).

The results, as shown in Table 2, fail to support Hypothesis 1 that physician and hospital resources are positively associated with discharge rates. Considering physician resources, the number of primary care physicians in the community (SPECPCMD) is positively associated with three medical and one surgical MDRGs: acute myocardial infarction, cardiac arrhythmia, angina pectoris, and major cardiovascular operations. SPECPED, the number of medical and pediatric specialists in the community, is negatively associated with two MDRGs: adult simple pneumonia and specific cerebrovascular disorders. SPECSURG or the number of general surgeons and surgical specialists is negatively associated with three MDRGs: adult bronchitis and asthma, heart failure and shock, and angina pectoris. SPECHOSP, the number of hospital-based specialists is negatively associated with four MDRGs: infectious disease disorders, major cardiovascular operations, gall bladder disease with cholecystectomy, and back and neck operations. In sum, significance is found for 13 or 19.6 percent of 56 coefficients. Of these 13 coefficients, 4 have positive signs as predicted in Hypothesis 1. 
With regard to hospital resources, the number of hospital beds in the market (BED) is not significantly associated with any MDRGs. The average number of unique services provided by hospitals in the community (M_DIF) is related to two MDRGs, adult gastroenteritis and major cardiovascular operations, but in opposite directions. The average number of registered nurses per bed in community hospitals (M_RN) is significantly associated with three MDRGs, heart failure and shock, major cardiovascular operations, and back and neck operations, but these effects are not consistent in their direction. The average level of hospital outpatient activity (M_OA) is positively related to both gall bladder disease with cholecystectomy and back and neck operations. The average percentage of primary care physicians on staff has statistically significant relationships with both chemotherapy and infectious-disease discharges but, again, in opposite directions. In sum, out of 70 coefficients, only 9 are statistically significant, and only 5 are in the predicted direction.

When physician and hospital resource capacity are considered together, 22 out of 126 coefficients (17.5 percent) are significant. The direction of these relationships offers little support to the general hypothesis; of 22 significant coefficients, only 9 are positive (7.1 percent of the total 126). Among the statistically significant effects, we could identify no systematic trends that would help interpret these findings. No clear differences separate the medical from surgical MDRGs and some of the capacity variables have negative effects on some MDRGs and positive effects on other MDRGs.

Hypothesis 2, which predicts that greater differentiation in physician and hospital resources is positively associated with hospital discharges across HMCs, receives no support from the results in Table 2. Two variables measure differentiation in physician resources: the percentage physicians who practice allopathic medicine (PCMD) and a diversity index of seven specialty areas (DVSTY). For hospital resources, differentiation is measured by the coefficient of variation in the number of services provided by a given hospital that are not available in other hospitals in the market (CV_DIF), the coefficient of variation of registered nurse staffing (CV_RN), the coefficient of variation of hospital outpatient activity (CV_OA), and the coefficient of variation of average percentage of primary care physicians on staff (CV_PC).

Among the differentiation variables, the coefficient of variation for the index of services provided across hospitals is not significant for any MDRGs. The other four variables, including the percentage of physicians practicing allopathic medicine (PCMD) and the coefficients of variation for nurse staffing (CV_RN), outpatient activity (CV_OA), and primary care physician staffing (CV_PC) are significant for only 1 out of 14 MDRGs each. In other words, among the physician and hospital differentiation, the analysis, which could 
have produced 84 significant coefficients, yields only 4 (4.8 percent) that are statistically significant. We conclude that Hypothesis 2 is not supported by these data and that more differentiated HMCs do not have noticeably higher hospital use than less differentiated HMCs.

Finally, Hypothesis 3 argues that greater teaching intensity, as measured by the number of FTE medical residents and interns per 1,000 population (FTERES), is associated with higher hospital discharges across HMCs. FTERES is significantly associated with three of the four surgical MDRGs and only 1 of the 10 medical MDRGs; however, the relationships are all negative, contrary to our hypothesis.

\section{IMPACT OF SOCIOECONOMIC AND TIME CONTROLS}

The general pattern of effects pertaining to SES and percentage African Americans in the HMC remains even after introducing the hospital and physician resource variables. Twelve of 28 socioeconomic variables are significant and, with one exception, in the same direction as in the baseline model. This indicates that socioeconomic effects on hospital use are not explainable by differences in hospital and physician resources.

Another important feature of the study is that, unlike most other SAV analyses, we use pooled cross-sectional time-series rates. Therefore, we are able to assess how MDRG discharge rates change over time. Results show that time is an important variable to control (Table 2). Compared to the reference year of 1986, significant time effects appear in 10 of the 14 MDRGs examined, either positively or negatively. For example, the discharge rates of adult gastroenteritis, adult bronchitis, and adult simple pneumonia generally decrease during the study period. On the other hand, three of the four surgical MDRGs and three medical MDRGs-infectious-disease disorders, specific cerebrovascular disorders, and heart failure and shock-show increasing discharge rates. Inpatient gallbladder surgery also increases during the study period, but such increase is accounted for by physician resource variables (apparently hospital-based specialists and house officers).

\section{CONCLUSION}

\section{KEY FINDINGS}

The central conclusion of this study is that during the period 1986-1992 in the lower peninsula of Michigan, socioeconomic variables had more frequent and consistent impact on hospital discharge rates than the hospital and physician resource characteristics examined in the study. These findings are 
important because much of the research on SAV has not included explicit controls for socioeconomic variables.

Second, we found no clear or consistent relationship between the hospital and physician resource variables and hospital discharge rates for 14 common and highly variable groups of clinical conditions, both medical and surgical. This conclusion holds when controlling for socioeconomic variables, market seepage to neighboring states, and for changes in discharge rates over time. In short, there is only weak evidence that the hospital and physician resource characteristics used in this study have meaningful associations with hospital market area variation.

The capacity hypothesis was supported only in one counterintuitive case- the number of acute myocardial infarction discharges increased with a larger supply of primary care physicians. We frankly cannot explain this relationship. Angina and cardiac surgery discharges also showed an increase, but the relationship was only marginally significant $(p<0.10)$. Interestingly, several discharge rates were diminished by increased presence of specialties, but the pattern was not relevant to the specialties involved. It is unclear why a higher number of medical specialties in the market community resulted in fewer cerebrovascular discharges, more surgeons reduced the rate of angina discharges, and more hospital-based physicians reduced the rate of cardiac surgery. One possibility is that norms of medical practice that reduce inappropriate use are more salient in hospital market communities with a higher number of medical specialties. Although this may explain the specific findings associated with these medical and surgical diagnoses/procedures, what remains problematic is why such patterns do not appear consistently across the 14 MDRGs examined in this study.

The differentiation hypothesis and the teaching-intensity hypothesis received no support from the findings. The level of teaching intensity was significantly associated with four MDRG discharges but in the direction opposite to that predicted. It is plausible that hospital markets with more house officers tend to perform more standardized procedures, admit fewer inappropriate gastroenteritis patients, and are more rigorous in their criteria for surgery.

\section{CAVEATS}

Our findings may be affected by the limited measures of market-level resources, particularly those related to differentiation. For example, other health services resources not explicitly measured in our study may affect the extent to which HMCs are differentiated and, in turn, hospital use. These resources might include primary care centers, nursing homes, nurse practitioners, or nurse midwives. To the extent that such resources matter, tests of 
our hypotheses are imperfect. This scenario seems plausible given the increasingly interdependent nature of health services delivery. A second potential measurement problem lies in our use of a dummy variable representing border HMCs to capture cross-border patient flow. At best, this measure accounts only for those areas at greatest risk of such flow and does not directly measure this phenomenon, particularly in other parts of the state that might also experience it.

A second limitation is that the findings refer to the major part of one state: the lower peninsula of Michigan. Generality beyond the boundaries of Michigan is therefore not formally justified. However, the size of the database, the longitudinal nature of the analysis, and a study design that both builds on and extends previous work provide a strong test of the relative effects of marketlevel hospital and physician resources versus socioeconomic factors on hospital use.

A third limitation is that our study period ends in 1992, and it is possible that more recent data would yield different results. This possibility cannot be ignored especially given the significant time effects that we have demonstrated and the emergence of managed care as a potent market force. However, even with time effects present, physician and hospital effects did not become more salient over time, nor did the socioeconomic effects diminish. These patterns suggest that more recent data would yield similar results, and, once again, our research is a guide for such efforts.

Notwithstanding these limitations, we believe that the results of this study stand as a valid empirical test of several important questions in SAV research. The large size of the sample, the use of multiple clinical conditions and 7 years of data, the statistical procedure used to remove the effects of autocorrelation, and the explicit inclusion of socioeconomic variables all sum to a stronger method than that of most studies on the subject.

\section{IMPLICATIONS}

Our thesis, which seemed reasonable at the outset, was that physician and hospital resources had important, direct effects on hospital use. Lower levels of hospital and physician resources, more homogeneity in those resources, and an emphasis away from teaching intensity in a community were expected to be associated with reduced hospital use. These are not new ideas; substantial efforts have been mounted to plan the health care establishment (Salkever and Bice 1976), to centralize management in larger organizations (Shortell et al. 1996), and to emphasize primary care (Vogel 1993). Our results, however, suggest that the world is not so simple. 
First, the inclusion of market-level socioeconomic factors in analyses of SAV seems critical. Although variation from community to community in the use of health services is often so great that the temptation to examine only characteristics of practice patterns is high, the present research concludes otherwise, a conclusion also reached by Bindman et al. (1995) and Komaromy et al. (1996). The key policy implication of these findings is that reducing the use of services may be as much as altering lifestyle and patterns associated with socioeconomic status and race, or the underlying socioeconomic foundations of a community. For example, if higher use of health services in low-SES communities can be traced to features of lifestyle and behavior, then health policy makers may need to fashion health education, health promotion, and other interventions that reduce whatever high use of services is implicated. Alternatively, if lower use of health services in, say, communities with a high proportion of African Americans is a function of factors related to poor access, such as lack of health insurance coverage, or to general economic deprivation, then efforts need to be directed toward improving access or enhancing economic development. It is unlikely, however, that individual institutions (e.g., health care systems) are well equipped to respond to these socioeconomic imperatives. Even when they are dominant providers in their market, these organizations will likely need to engage in community-wide efforts with other partners to systematically address prioritized targets. In so doing they will diffuse the risk of these systemic efforts and increase receptivity among the target groups (Griffith 1998).

Our findings suggest that hospital and physician impact may be either deeper than the available measures can reveal or weaker in their effects than many have proposed. Notions of health facility consolidation, institutional collaboration, and physician retraining, while they certainly have a place in health policies, may not effectively correct the underlying forces (e.g., community economic development, poverty, education) that influence health service utilization. Benchmarking, goal setting, premium setting, and proposals for corrective action that do not recognize this fact will have only limited results. In particular, caution must be exercised in assuming that the lowest, or even the average, level of use should be a normative target in cost control schemes founded on benchmarking. Before such efforts are undertaken, research should determine how much of the particular use of services is a function of the disease patterns, and demographic and socioeconomic characteristics of the area before defining a desirable level of use and implementing activities to achieve that goal.

At the same time, our study suggests that the causes of high use are not uniform, and the solutions to address such use must similarly be tailored to the 
natural history of each disease. This may lead to increases in some conditions as access barriers are removed. Finally, the issue of adverse selection looms large. Our data suggest that the key factors in hospital use are population characteristics, not the physician panel or institutions responsible for care management in the market. Organizations and physician panels operating in markets with higher risk populations can easily be mislabeled as inefficient, while those in favored settings may mask real inefficiencies under unacknowledged population differences.

As managed care continues to emphasize cost and utilization controls and evolves to also consider disease prevention and health promotion, it will become clear that the primary point of leverage for affecting health may not be health services resources such as physicians and hospitals but rather the socioeconomic context of the communities in which individuals work and live. Indeed, our findings provide some preliminary evidence that the socioeconomic makeup of communities represents a key determinant of marketlevel health care use. Without consideration of such context, changes to the structure of the health services system may have little impact on such use.

\section{REFERENCES}

Billings, J., L. Zeitel, J. Lukomnik, T. S. Carey, A. E. Blank, and L. Newman. 1993. Impact of Socio-Economic Status on Hospital Use in New York City. Health Affairs 12 (Spring): 162-73.

Bindman, A. B., K. Grumbach, D. Osmond, M. Komaromy, K. Vranizan, N. Lurie, J. Billings, and A. Stewart. 1995. Preventable Hospitalizations and Access to Health Care. Journal of the American Medical Association 274 (July): 305-11.

Breslow, N. E., and N. E. Day. 1987. Statistical Methods for Cancer Research. Vol. 2. Lyon, France: International Agency for Research on Cancer.

Cain, K. C., and P. Diehr. 1992. Testing the Null Hypothesis in Small Area Analysis. Health Services Research 27:267-94.

Carlisle, D. M., R. B. Valdez, R. B. Shapiro, and R. H. Brook. 1995. Geographic Variation in Rates of Selected Surgical Procedures within Los Angeles County. Health Services Research 30:27-42.

Dartmouth Center for the Evaluative Clinical Sciences. 1998. The Dartmouth Atlas of Health Care in the United States 1998. Chicago: American Hospital Publishing.

Escarce, J. J. 1993. Would Eliminating Differences in Physician Practice Style Reduce Geographic Variations in Cataract Surgery Rates? Medical Care 31 (December): 1106-18.

Evan, W. R. 1967. The Organization Set: Toward a Theory of Interorganizational Relations. In Approaches to Organization Design, ed. D. T. James; 173-91. Pittsburgh: University of Pittsburgh Press. 
Fennell, M. L. 1980. The Effects of Environmental Characteristics on the Structure of Hospital Clusters. Administrative Science Quarterly 21:485-510.

- 1982. Context in Organizational Groups: The Case of Hospital Clusters. Administrative Science Quarterly 23:65-84.

Folland, S., and M. Stano. 1990. Small Area Variations: A Critical Review of Propositions, Methods, and Evidence. Medical Care Review 47:419-65.

Gittelsohn, A. M., J. Halpern, and R. L. Sanchez. 1991. Income, Race, and Surgery in Maryland. American Journal of Public Health 81 (November): 1435-41.

Gittelsohn, A., and N. R. Powe. 1995. Small Area Variations in Health Care Delivery in Maryland. Health Services Research 30:295-317.

Goodman, D. C., E. S. Fisher, A. Gittelsohn, C. H. Chang, and C. Fleming. 1994. Why Are Children Hospitalized? The Role of Non-Clinical Factors in Pediatric Hospitalizations. Pediatrics 93 (June): 896-902.

Goodman, D. C., and G. R. Green. 1996. Assessment Tools: Small Area Analysis. American Journal of Medical Quality 11:S12-S14.

Gottlieb, D. J., A. S. Beiser, and G. T. O'Connor. 1995. Poverty, Race, and Medication Use Are Correlates of Asthma Hospitalization Rates. A Small Area Analysis in Boston. Chest 108 (July): 28-35.

Green, L. A. 1996. Practical Issues in Conducting Small-Area Variation Analysis. Family Medicine 28:277-81.

Green, L. A., and M. P. Becker. 1994. Physician Decision Making and Variation in Hospital Admission Rates for Suspected Acute Cardiac Ischemia. A Tale of Two Towns. Medical Care 32 (November): 1086-97.

Griffith, J. R. 1998. Designing 21st Century Healthcare: Leadership in Hospitals and Health Systems. Chicago: Health Administration Press.

Griffith, J. R., J. D. Restuccia, P. J. Tedeschi, P. A. Wilson, and H. S. Zuckerman. 1981. Measuring Community Hospital Services in Michigan. Health Services Research 16:135-73.

Hannan, M. T., and A. A. Young. 1977. Estimation in Panel Methods: Results on Pooling Cross-Sections and Time Series. In Sociological Methodology 1977, ed. D. R. Heise; 52-83. San Francisco: Jossey-Bass.

Hermann, R. C., R. A. Dorwart, C. W. Hoover, and J. Brody. 1995. Variation in ECT Use in the United States. American Journal of Psychiatry 152 (6): 869-75.

Hine, C., V. A. Wood, S. Taylor, and M. Charny. 1996. Do Community Hospitals Reduce the Use of District General Hospital Inpatient Beds? Journal of the Royal Society of Medicine 9 (12): 681-87.

Iglehart, J. K., ed. 1984. Variations in Medical Practice. Health Affairs 3:1-160.

Joseph, C. L., S. L. Havstad, D. R. Ownby, C. C. Johnson and B. C. Tilley. 1998. Racial Differences in Emergency Department Use Persist despite Allergist Visits and Prescriptions Filled for Antiinflamatory Medications. Journal of Allergy and Clinical Immunology 101 (4): 484-90.

Komaromy, M., N. Lurie, D. Osmond, K. Vranizan, D. Keane, and A. B. Bindman. 1996. Physician Practice Style and Rates of Hospitalization for Chronic Medical Conditions. Medical Care 34 (June): 594-609. 
Kovac, S. R., S. J. Christie, and G. A. Bindbeutel. 1991. Abdominal vs. Vaginal Hysterectomy: A Statistical Model for Determining Physician Decision Making and Patient Outcome. Medical Decision Making 11:19-28.

Kuhn, E. M., A. J. Hartz, and M. Baras. 1995. Correlation of Rates of Coronary Artery Bypass Surgery. Health Services Research 30:425-36.

Liang, K. Y., and R. K. Zeger. 1986. Longitudinal Data Analysis Using Generalized Linear Models. Biometrika 73:13.

Luft, H. S., and B. W. Brown, Jr. 1993. Calculating the Probability of Rare Events: Why Settle for an Approximation? Health Services Research 28 (October): 419-39.

Luft, H. S., J. C. Robinson, D. W. Garnick, S. C. Maerki, and S. J. McPhee. 1986. The Role of Specialized Clinical Services in Competition among Hospitals. Inquiry 23 (1): 83-94.

Lundberg, G. D., and J. E. Wennberg. 1997. A JAMA Theme Issue on Quality of Care. A New Proposal and a Call to Action. JAMA 278 (19): 1615-16.

Malec, D., and J. Sedransk. 1993. Bayesian Predictive Inference for Units with Small Sample Sizes. The Case of Binary Random Variables. Medical Care 31 (May): YS66-70.

McMahon, L. F. Jr., R. A. Wolfe, J. R. Griffith, and D. Cuthbertson. 1993. Socioeconomic Influence on Small Area Hospital Utilization. Medical Care 31 (May): YS29-36.

Office of Health and Medical Affairs. 1990. The OHMA Physician Census: Introduction and Technical Notes. Lansing: Michigan Department of Management and Budget, Office of Health and Medical Affairs.

Paul-Shaheen, P., J. D. Clark, and D. D. Williams. 1987. Small Area Analysis: A Review and Analysis of the North American Literature. Journal of Health Politics, Policy and Law 12:741-809.

Restuccia, J., M. Shwartz, A. Ash, and S. Payne. 1996. High Hospital Admission Rates and Inappropriate Care. Health Affairs 15 (Winter): 156-63.

Rice, T. H. 1983. The Impact of Changing Medicare Reimbursement Rates on Physician Induced Demand. Medical Care 21:803-15.

Roos, N. P., C. D. Black, L. L. Roos, R. B. Tate, and K. C. Carriere. 1995. A PopulationBased Approach to Monitoring Adverse Outcomes of Medical Care. Medical Care 33 (February): 127-38.

Salkever, D. S., and T. W. Bice. 1976. The Impact of Certificate-of-Need Controls on Hospital Investment. Milbank Memorial Fund Quarterly Health Sociology 54 (2): 185-214.

Scott, W. R. 1992. Health Care Organizations in the 1980s: The Convergence of Public and Professional Control Systems. In Organizational environments: Ritual and Rationality, ed. J. W. Meyer and W. R. Scott; 99-113. Newbury Park, CA: Sage.

Shortell, S. M., R. Gillies, D. Andersen, K. Morgan-Erickson, and J. Mitchell. 1996. Remaking Health Care in America. San Francisco: Jossey-Bass.

Shwartz, M., A. S. Ash, J. Anderson, L. I. Iezzoni, S. M. Payne, and J. D. Restuccia. 1994. Small Area Variations in Hospitalization Rates: How Much You See Depends on How You Look. Medical Care 32 (March): 189-201.

Sofaer, S., and R. C. Myrtle. 1991. Interorganizational Theory and Research: Implications for Health Care Management, Policy, and Research. Medical Care Review 48:371-409. 
Stano, M. 1986. A Further Analysis of the "Variation in Practice Style" Phenomenon. Inquiry 23:176-82.

- 1991. Further Issues in Small Area Variation Analysis. Journal of Health Policy, Politics \& Law 16:573-88.

Tape, T. G., P. S. Heckerling, J. P. Ornato, and R. S. Wigton. 1991. Use of Clinical Judgment Analysis to Explain Regional Variations in Physician's Accuracy in Diagnosing Pneumonia. Medical Decision Making 11:189-97.

Teachman, J. D. 1980. Analysis of Population Diversity: Measures of Qualitative Variation. Sociological Methods and Research 8:341-362.

Thomas J. W., J. R. Griffith, and P. Durance. 1980. Defining Hospital Clusters and Associated Service Communities in Metropolitan Areas. Journal of Socio-Economic Planning Sciences 15:45-51.

Turk, H. 1977. Organizations in Modern Life. San Francisco: Jossey-Bass.

Vogel, D. E. 1993. The Physician and Managed Care. Chicago: American Medical Association.

Volinn, R., P. Diehr, M. A. Ciol, and J. D. Loeser. 1994. Why Does Geographic Variation in Health Care Practice Matter? (And Seven Questions to Ask in Evaluating Studies on Geographic Variation.) Spine 19:2092S-2100S.

Wennberg, J. E. 1984. Dealing with Medical Practice Variations: A Proposal for Action. Health Affairs 3:6-32.

Wennberg, J. E., J. L. Freeman, and W. J. Culp. 1987. Are Hospital Services rationed in New Haven or Over-Utilized in Boston? Lancet 23:1185-88.

Wennberg, J., and A. Gittelsohn. 1973. Small Area Variations in Health Care Delivery. Science 182 (December): 1102-8.

Wennberg, J. E., and A. Gittelsohn. 1982. Variations in Medical Care among Small Areas. Scientific American 246:120-34.

Wyatt, S. M., E. Moy, R. J. Levin, K. B. Lawton, D. M. Witter Jr., E. Valente Jr., R. Lala, and P.F. Griner. 1997. Patients Transferred to Academic Medical Centers and Other Hospitals: Characteristics, Resource Use, and Outcomes. Academic Medicine 72 (10): 921-30.

Zeger, R. K., and K. Y. Liang. 1992. An Overview of Methods for the Analysis of Longitudinal hospitals: Characteristics, Resource Use, and Outcomes. Academic Medicine 72 (10): 921-30. Data. Statistics in Medicine 11:1825-39. 